\title{
1 A statistical shape model to predict the 2 premorbid glenoid cavity
}

3 Daniel Abler ${ }^{1}, \mathrm{PhD}$, Steve Berger ${ }^{1}, \mathrm{PhD}$, Alexandre Terrier ${ }^{2}, \mathrm{PhD} \mathrm{Fabio} \mathrm{Becce}^{3}, \mathrm{MD}$,

$4 \quad$ Alain Farron ${ }^{4}, \mathrm{MD} \&$ Philippe Büchler $^{1}, \mathrm{PhD}$

$5 \quad{ }^{1}$ Institute for Surgical Technology and Biomechanics, University of Bern, Bern, Switzerland

$6{ }^{2}$ Laboratory of Biomechanical Orthopedics, Ecole Polytechnique Fédérale de Lausanne,

7 Lausanne, Switzerland; ${ }^{3}$ Department of Diagnostic and Interventional Radiology, Lausanne

8 University Hospital, Lausanne, Switzerland; ${ }^{4}$ Service of Orthopedics and Traumatology,

9 Lausanne University Hospital, Lausanne, Switzerland

10 Contributor responsible for the manuscript and proofs:

11 Prof. Dr. Philippe Büchler

12 Institute for Surgical Technology and Biomechanics, University of Bern, Stauffacherstrasse 78,

13 CH- 3014 Bern, Switzerland

14 philippe.buechler@istb.unibe.ch

15 Disclaimer: No outside funding or grants was received for this study.

16 IRB: Commission cantonale d'éthique (CER-VD), Protocol 136/15

17 Short title: Glenoid prediction using statistical shape modeling

18 Total word count: 3162 
Glenoid prediction using statistical shape modeling

\section{Abstract}

20 Background: This study proposes a method for inferring the premorbid glenoid shape and

21 orientation to inform restorative surgery.

22 Methods: We developed a statistical shape model (SSM) from 64 healthy scapulae. The premorbid 23 glenoid shape was predicted from the surrounding scapular body using a SSM-based

24 reconstruction method. First, the method was validated on 64 healthy scapulae by quantifying the 25 accuracy of the predicted surface in terms of surface distance, as well as glenoid version and 26 inclination. The SSM-based reconstruction was then applied to 30 scapulae with OA glenoids.

27 Glenoid version and inclination were measured fully automatically and compared between the 28 original OA glenoids, SSM-based glenoid reconstructions, and healthy scapulae.

29 Results: Validation on healthy scapulae showed a root mean square surface distance between 30 original and predicted glenoid cavities of $1.0 \mathrm{~mm} \pm 0.2 \mathrm{~mm}$. The prediction error was $2.1^{\circ} \pm 1.6^{\circ}$ for

31 glenoid version and $2.1^{\circ} \pm 1.8^{\circ}$ for inclination. Differences between original and predicted glenoid 32 measurements were not statistically significant ( $\mathrm{p} \geq 0.42)$. When applied to OA dataset, SSM-based 33 reconstruction restored the glenoid version and inclination to values similar to the healthy 34 situation. No differences were observed between SSM-based reconstructed glenoids and healthy 35 scapulae ( $\mathrm{p} \geq 0.44$ ), while reconstructed glenoids significantly differed from original scapulae with 36 OA glenoids $(\mathrm{p} \leq 0.03)$.

37 Conclusion: The proposed local SSM can accurately predict the premorbid glenoid cavity of 38 healthy scapulae from anatomic features regarded as unaffected by degeneration. This technique 
Glenoid prediction using statistical shape modeling

39 has the potential to reconstruct the premorbid glenoid cavity as it was prior to OA, and thus guide

40 the orientation of glenoid implants in total shoulder arthroplasty.

41 Level of evidence: Basic Science Study; Computer Modeling

42 Keywords: Glenoid, Total Shoulder Arthroplasty, Computed Tomography, 3D Reconstruction, 43 Statistical Shape Model, Version, Inclination

44 
Glenoid prediction using statistical shape modeling

\section{Introduction}

46 Patients suffering from shoulder osteoarthritis (OA) may exhibit severe wear of their glenohumeral

47 joint $^{9}$ and various deformity patterns of the glenoid cavity ${ }^{1,10,17}$. When nonsurgical treatments fail,

48 shoulder arthroplasty can be performed to replace the degenerated glenohumeral joint by

49 prostheses. Accurate positioning of the glenoid component is crucial for the long-term success of

50 total shoulder arthroplasty ${ }^{15}$ as incorrect positioning can lead to glenoid implant failure and/or

51 glenohumeral subluxation and/or dislocation ${ }^{5,7}$. When glenohumeral OA is unilateral, the patient-

52 specific orientation of the glenoid implant might be determined based on the healthy contralateral

53 shoulder. However, glenohumeral OA is often bilateral so that the original orientation, size, and

54 shape of the glenoid cavity is usually unknown at the time of surgery.

55 Different techniques have been proposed to correct pathologic glenoid version due to $\mathrm{OA}^{6,25}$. One

56 of these approaches relied on positioning a three-dimensional reference model of the glenoid vault

57 on the endosteal surface of the pathologic bone ${ }^{25}$. More recently, this method has been compared

58 to predictions of the glenoid orientation based on landmarks located on the body of the scapula. A

59 linear relation was found between the anterior glenoid wall angle - defined by these landmarks -

60 and the glenoid version of healthy scapulae ${ }^{6}$. These previous studies indicate that a correlation

61 exists between healthy glenoid anatomy and the morphology of structures unaffected by OA,

62 which may allow predicting healthy glenoid version. However, such correlation alone provides no

63 information about the glenoid's three-dimensional shape or orientation. 
Glenoid prediction using statistical shape modeling

64 Statistical shape modeling (SSM) is a method that identifies the average and principal variations

65 of shape within a training population. One strength of this method is its ability to extrapolate a

66 complete anatomic surface representation from a sparse set of spatial positions. For example, SSM

67 has been used previously to predict the complete shape of the human femur from a sparse set of

68 landmarks collected on its proximal surface ${ }^{24}$ or to predict the premorbid shape of the proximal

69 humerus $^{23}$. Recently, SSM has also been proposed for the reconstruction of glenoid bone defects ${ }^{22}$.

70 While the authors showed that this approach accurately reconstructs the shape of healthy scapulae,

71 they did not evaluate the performance of this method on scapulae affected by OA.

72 Similar to this recent study ${ }^{22}$, we have developed an SSM-based approach for reconstructing the

73 "healthy" surface of glenoid cavities affected by OA. Instead an SSM of the entire scapula, we

74 propose using a local SSM, centered around the glenoid bone. Our first objective was to quantify

75 the accuracy of SSM-based predictions on a population with no signs of scapula deformation by

76 comparing the glenoid cavity predicted by the SSM to the actual three-dimensional glenoid

77 anatomy. The second objective of this work was to evaluate the ability of the SSM model to predict

78 the premorbid glenoid cavity and its orientation for patients exhibiting different patterns of 79 glenohumeral OA. 
Glenoid prediction using statistical shape modeling

\section{Materials \& Methods}

81 Our study was performed in three main steps. First, a 'local' SSM was created from a dataset of

82 healthy scapulae (i.e. with no signs of pathology on computed tomography (CT)), which was used

83 to reconstruct glenoid shape based solely on information from the bony regions surrounding it

84 (Fig. 1). In a second step, the SSM-based prediction method was tested on healthy scapulae for

85 validation. Finally, the method was used to predict the premorbid shape of scapulae with glenoids

86 affected by OA.

\section{$87 \quad$ Statistical Shape Model}

88 A dataset of 64 healthy scapulae (42 men, 22 women, between 17 and 88 years of age, average

89 age of 59.6 years) was used in this IRB-approved study (protocol 136/15). Consecutive whole-

90 body CT scans (64-detector row CT system) of polytrauma patients were retrospectively reviewed

91 by an experienced musculoskeletal radiologist. Patients with CT signs of glenohumeral OA,

92 glenoid dysplasia, scapular fracture, previous shoulder surgery, motion or any other CT artifacts,

93 and incomplete coverage of the scapulae were excluded. CT images of these scapulae were

94 subsequently segmented manually, and models of their 3D shape were generated as surface

95 meshes. Models of left scapulae were mirrored to resemble the configuration of right scapulae.

96 The positions of all corresponding anatomic landmarks were established automatically for all the

97 bones in the dataset ${ }^{18,19}$. With this information, the average healthy scapula shape and its modes

98 of variations could be computed ${ }^{14}$, defining the SSM and describing how anatomic landmarks 
Glenoid prediction using statistical shape modeling

99 move together when the shape of the scapula changes (Fig. 2). This study used a 'local' SSM that

100 did not include the entire scapula, but only the region of the glenoid as shown in Figure 1.

101 Validation on Healthy Scapulae

102 The shape of the healthy glenoid was reconstructed by fitting the SSM to the scapula region 103 surrounding the glenoid on the target shape (Fig. 1). The fitting was performed by adjusting the 104 contribution of each mode of variation of the SSM until the predicted shape best matched the shape 105 of the target bone in the region for fitting (Fig. 1, red surface). The SSM describes how the 106 proportions of different parts of the shape are linked. Therefore, the parameter set that optimally 107 fits the bone surrounding the glenoid also simultaneously provides a prediction of the glenoid 108 cavity. Since the SSM was built from healthy scapulae, the predicted glenoid cavity has the 109 characteristics of a healthy or premorbid glenoid. Thus, this approach provides an approximation 110 of healthy glenoid shape for the particular scapula used as fitting target.

111 The SSM creation and prediction process was repeated for each of the 64 scapulae in a leave-one112 out analysis. In each repetition, one scapula was selected and the SSM was built from the remaining 11363 scapulae. This SSM was then used to predict the overall surface of the scapula that had been 114 "left out" from the SSM creation process (Fig. 1).

115 A semi-automated CT measurement method was adapted and fully automatized to quantify glenoid 116 version, inclination, and medialization in a standardized and reproducible way ${ }^{27}$. The original 117 semi-automated method uses 11 bony landmarks that are placed manually on the non-eroded 
118 scapula to define a coordinate system in relation to which glenoid characteristics can be quantified.

119 Our fully automatized approach leverages the SSM to transfer the positions of these landmarks 120 automatically from the surface model of a single scapula to the surface models of all individual 121 scapula $^{21}$. As the resulting landmark positioning tends to be slightly inaccurate in practice, the 122 positions of the landmarks were automatically corrected based on the local curvature of the 123 scapula, following the same criteria as for manual landmark placement. ${ }^{27}$ The resulting scapula 124 coordinate system was then used to quantify glenoid version, inclination, and medialization.

125 Accuracy of predictions was evaluated in each repetition of the leave-one-out analysis. The quality 126 of shape prediction was quantified using the distance between the reconstructed glenoid cavity and 127 the surface of the bone obtained from its segmentation. We report the root mean square (RMS) 128 distance error for the glenoid cavity and the entire predicted shape, which includes the bony regions 129 used for the fitting process. The difference between measurements of the original and 130 reconstructed glenoids was evaluated using Bland-Altman plots. Statistical significance $(\mathrm{p}<0.05)$

131 was checked with paired Student's t-tests. Average cohort results were reported as mean \pm standard 132 deviation of absolute (unsigned) difference between original and reconstructed measures.

\section{Premorbid Shape of OA Glenoids}

134 Preoperative nonarthrographic shoulder CT scans of 30 patients who underwent anatomic total 135 shoulder arthroplasty for primary glenohumeral OA were randomly selected from our institutional 136 database (18 men, 12 women, between 54 and 88 years of age, average age of 71 years). Patterns 137 of glenoid OA were classified according to the updated Walch grading system ${ }^{1}$ by the same 
Glenoid prediction using statistical shape modeling

138 musculoskeletal radiologist, in consensus with an experienced shoulder surgeon (Table I). The 139 scapulae were manually segmented, and models of their 3D shape were generated as surface 140 meshes.

141 Prediction of the premorbid shape followed the same procedure as for the validation study on 142 healthy scapulae. Glenoid version, inclination, and medialization were quantified by the fully 143 automatized measurement method described previously. Differences in these measurements 144 between the original scapulae with OA and the reconstructed premorbid shapes were statistically 145 evaluated using paired Student's t-tests; differences to healthy glenoids were assessed using two 146 sample Student's t-tests. The significance level set was at $\mathrm{p}<0.05$. 
Glenoid prediction using statistical shape modeling

147 Results

148 Statistical Shape Model

149 The SSM was built from all healthy scapulae of the training dataset. The first 3 modes of variation

150 represented more than $60 \%$ of the overall shape variability across subjects (Fig. 2), while less than

15120 modes of the SSM explained about $95 \%$ of shape variability. Although the modes of variation

152 do not have direct morphologic interpretation, the first few modes of the scapula SSM displayed

153 characteristic features: the first mode of variation was associated with a change in size, the second

154 mode predominantly reflected a change in angle between the acromion and the coracoid, while the

155 third mode was related to variations in glenoid inclination (Fig. 2).

156 Validation on Healthy Scapulae

157 The leave-one-out analysis for evaluating glenoid reconstruction accuracy showed that the SSM 158 accurately reproduces the surface of the glenoid region (Fig. 3). On average, the RMS 159 reconstruction error was slightly above $0.5 \mathrm{~mm}$ on the bone surface used for fitting $160(0.6 \mathrm{~mm} \pm 0.1 \mathrm{~mm})$. The reconstruction error was larger for the predicted glenoid cavity, yet its 161 average error remained at $1 \mathrm{~mm}(1.0 \mathrm{~mm} \pm 0.2 \mathrm{~mm})$. Figure 3 shows the spatial distribution of surface

162 distance between original and SSM-based reconstructed glenoids. Surface distance of the subjects

163 with the smallest (Fig. 3b) and largest (Fig. 3c) reconstruction error on the glenoid cavity were

164 compared to the average surface distance across all samples (Fig. 3a). Surface distance remained 165 below $1 \mathrm{~mm}$ on most of the reconstructed surface, yet could reach up to approximately $3.5 \mathrm{~mm}$ on 166 the superior aspect of the glenoid rim in the worst fitting sample. 
Glenoid prediction using statistical shape modeling

167 Heathy scapulae included in this study had a glenoid version of $8.1^{\circ} \pm 5.2^{\circ}$, inclination of $8.6^{\circ} \pm 5.3^{\circ}$,

168 and medialization of $19.2 \mathrm{~mm} \pm 1.8 \mathrm{~mm}$. The average absolute difference between AAM-based

169 reconstructed and the healthy reference glenoids was $2.1^{\circ} \pm 1.6^{\circ}$ for glenoid version, $2.1^{\circ} \pm 1.8^{\circ}$ for

170 inclination, and $0.7 \mathrm{~mm} \pm 0.5 \mathrm{~mm}$ for medialization, with $95 \%$ confidence intervals between $-5.2^{\circ}$

171 and $5.3^{\circ}$ for glenoid version, $-5.5^{\circ}$ and $5.4^{\circ}$ for inclination, and $-1.7 \mathrm{~mm}$ and $1.8 \mathrm{~mm}$ for

172 medialization (Fig. 4). The prediction error was almost independent of the magnitude of the

173 respective measurements. No significant differences were found between the glenoid version

$174(p=0.93)$, inclination $(p=0.97)$, and medialization $(p=0.42)$ of the SSM-based reconstructed and

175 healthy reference glenoids.

\section{Premorbid Shape of OA Glenoids}

177 The shape of the premorbid glenoid was reconstructed for each of the 30 OA samples. Overall, the

178 SSM-based reconstruction restored glenoid orientation with values of version and inclination close

179 to the healthy situation (Fig. 5). Statistical analysis revealed no difference between SSM-based

180 reconstructed glenoid version and the healthy situation $(\mathrm{p}=0.97)$, whereas the version of the

181 original OA glenoids was significantly different from the version of the SSM-based reconstructed

182 glenoids $(\mathrm{p}<0.01)$ and of the healthy situation $(\mathrm{p}<0.01)$. Similar results were obtained for the

183 glenoid inclination, where no difference was observed between SSM-based reconstructed and

184 healthy configurations $(\mathrm{p}=0.44)$, while the inclination of the original OA glenoids was significantly 185 different from both the SSM-based reconstructed $(p=0.03)$ and healthy $(p=0.02)$ scapulae (Fig. 5).

186 On the other hand, medialization was not significantly influenced by the SSM-based 187 reconstruction. 
Glenoid prediction using statistical shape modeling

188 Our OA dataset included samples of six different Walch classes (Table I). The model was able to 189 correct the posterior version for the B3, B2, and A2 OA glenoid types, with the largest correction 190 for B3 and lowest for A2 glenoids. On the other hand, the correction for the A1 and B1 glenoid 191 types was close to zero. SSM-based reconstruction increased the inclination for all Walch classes, 192 except for type D where a decrease of approximately $4^{\circ}$ was measured. Again, the medialization 193 was not affected by the reconstruction and remained constant. 
Glenoid prediction using statistical shape modeling

195 Discussion

196 Optimal glenoid implant positioning is challenging for patients suffering from glenohumeral OA

197 with substantial wear of the glenoid cavity. This study shows that an approach based on SSM

198 provides an accurate prediction of the entire three-dimensional surface of the healthy glenoid and

199 is able to reconstruct the premorbid glenoid orientation of pathologic scapulae.

200 Validation of the SSM-based technique on healthy scapulae indicates that the premorbid shape can

201 be reconstructed with millimeter accuracy. Our results (1.0mm RMS error) are similar to the data

202 recently reported by Plessers et al. ${ }^{22}$ using a SSM of the entire scapula shape (1.2mm RMS error).

203 Since clinical shoulder CT scans have a spatial resolution of the same magnitude, this level of

204 prediction accuracy is comparable with the accuracy by which a glenoid could be reconstructed in

$2053 \mathrm{D}$ from an actual CT dataset. The validation on healthy cases also showed that the glenoid version

206 can be predicted with an error around $2^{\circ}$, which is comparable to the results obtained by Plessers

207 et al. $^{22}$ using a SSM of the scapula $\left(2.9^{\circ}\right)$, but smaller than the prediction error of the glenoid vault

208 model proposed by Scalise et al. ${ }^{25}\left(3.7^{\circ}\right)$ and the anterior glenoid wall angle model developed by

209 Ganapathi et al. $^{6}\left(3.2^{\circ}\right)$. Overall, the precision by which the SSM model reproduced the healthy

210 glenoid orientation is of the same magnitude as the precision of glenoid implant positioning using

211 patient-specific guides, which has been reported between $1.8^{\circ}$ and $4.3^{\circ}$ for version and between

$2121.2^{\circ}$ and $3.1^{\circ}$ for inclination ${ }^{4,8,11,12}$.

213 Clinical applicability of the SSM-based glenoid reconstruction approach was demonstrated on 214 scapulae with various OA glenoid patterns. Prediction results of glenoid version and inclination 
215 followed the clinical intuition for each Walch class, applying a larger correction on the version

216 where the wear was larger and leaving the version unchanged otherwise. Statistically, predicted

217 glenoid version and inclination were compatible with characteristics of a healthy scapula dataset,

218 but significantly different from the original OA shapes. This evaluation on scapulae with OA

219 glenoids demonstrates that the proposed reconstruction process restores glenoid orientation.

220 Validation of the prediction accuracy on individual scapulae remains challenging as it requires

221 knowledge of the shape of the scapula prior to degradation by OA. Cases of unilateral

222 glenohumeral OA deformation provide a route for validating such studies; however, these are

223 relatively rare and difficult to obtain from routine CT data. Furthermore, it remains unclear if the

224 non-OA side is truly normal and if these patients had symmetric scapulae before pathologic

225 deformation $^{26}$. Therefore, a longitudinal dataset of shoulder CT scans would be needed, which

226 follows patients from the premorbid state to fully developed glenohumeral OA, eventually

227 requiring shoulder arthroplasty. Despite the difficulties to fully validate the proposed procedure

228 for reconstructing OA glenoids in individual patients, SSM-based prediction of shoulder anatomy

229 could also be used in various other clinical settings. For example, in cases of bony Bankart

230 fractures, the method could support the surgical decision-making process by pre-surgical

231 reconstruction of the premorbid glenoid position and orientation.

232 The measurement of glenoid version is traditionally based on a 2D approach using a specific axial

233 CT slice. This approach has been shown to be inaccurate as it depends on the position of the 234 scapula - and thus of the patient - as visualized on the CT slice, and does not account for bone 
Glenoid prediction using statistical shape modeling

235 erosions outside of the axial CT plane $e^{2,3,9,20}$. Several methods have been developed to take into

236 account the three-dimensional nature of the scapula anatomy ${ }^{6,13,16}$, yet their implementation in

237 clinical practice remains challenging and they are limited to a few anatomic measurements. On the

238 other hand, the SSM-based approach proposed here, not only predicts these clinical measurements,

239 but also the entire shape of the original glenoid cavity. Since the error of the surface reconstruction

240 is small, we believe that glenoid implant positioning based on the complete 3D shape will be more

241 robust and thus represents a better predictor for preoperative planning.

242 A preliminary analysis had shown that the prediction of the glenoid cavity was more accurate when

243 the SSM only included the glenoid, parts of the acromion and coracoid, instead of the entire

244 scapula. An additional advantage of this approach is that the tips of the acromion and coracoid can

245 be excluded from the model. These regions are difficult to segment from CT images and not always

246 fully included in the original CT field of view for radiation protection reasons. By excluding these

247 regions, we obtained improved prediction accuracy of healthy scapulae, while removing error-

248 prone surface information from the fitting process.

249 This study has limitations. First, the number of samples in each Walch class does not permit 250 conclusions about statistical significance by subgroup. However, the orientation correction 251 observed for each Walch class agrees with clinical intuition. Moreover, this technique allowed 252 restoring average version and inclination of glenoids affected by a representative range of OA253 induced deformations to values characteristic for the healthy population. A further limitation 254 concerns the size of the glenoid region that is expected to be affected by OA and therefore excluded 
Glenoid prediction using statistical shape modeling

255 from the fitting process (Fig. 1). We assumed that the osteoarthritic deformation was limited to the

256 area in close proximity to the glenoid cavity, but OA might affect other regions and extend to the

257 acromion and coracoid. Our results indicate that the proposed approach is suitable for correcting

258 OA-induced glenoid deformations, yet for future clinical application, SSM-based predictions

259 should be further evaluated.

260 Conclusions

261 This study shows that it is possible to rely on a local SSM to predict the three-dimensional shape

262 of the glenoid cavity with an average surface reconstruction error of $1 \mathrm{~mm}$, and the glenoid

263 orientation with a precision of approximately $2^{\circ}$. Moreover, glenoid version and inclination

264 reconstructed from OA datasets are statistically comparable with healthy scapulae. This approach

265 provides a quantitative method to help clinicians in preoperatively determining the glenoid implant

266 orientation in patients with glenohumeral OA. 
Glenoid prediction using statistical shape modeling

267

268

269

270

271

272

273

274

275

276

277

278

279

280

281

282

283

284

\section{References}

1. Bercik MJ, Kruse K, Yalizis M, Gauci M-O, Chaoui J, Walch G. A modification to the Walch classification of the glenoid in primary glenohumeral osteoarthritis using threedimensional imaging. J. Shoulder Elbow Surg. [Internet]. 2016 Jun 6;Available from: http://www.ncbi.nlm.nih.gov/pubmed/27282738doi:10.1016/j.jse.2016.03.010

2. Bokor DJ, O'Sullivan MD, Hazan GJ. Variability of measurement of glenoid version on computed tomography scan. J. shoulder Elb. Surg. [Internet]. 1999 Nov;8(6):595-8. Available from: http://www.ncbi.nlm.nih.gov/pubmed/10633895doi:10.1016/S10582746(99)90096-4

3. Budge MD, Lewis GS, Schaefer E, Coquia S, Flemming DJ, Armstrong AD. Comparison of standard two-dimensional and three-dimensional corrected glenoid version measurements. J. shoulder Elb. Surg. [Internet]. 2011 Jun;20(4):577-83. Available from: http://www.ncbi.nlm.nih.gov/pubmed/21324716doi:10.1016/j.jse.2010.11.003

4. Eraly K, Stoffelen D, Vander Sloten J, Jonkers I, Debeer P. A patient-specific guide for optimizing custom-made glenoid implantation in cases of severe glenoid defects: An in vitro study. J. Shoulder Elb. Surg. [Internet]. 2016;25(5):837-845. Available from: http://dx.doi.org/10.1016/j.jse.2015.09.034doi:10.1016/j.jse.2015.09.034

5. Farron A, Terrier A, Büchler P. Risks of loosening of a prosthetic glenoid implanted in 
Glenoid prediction using statistical shape modeling

285

286

287

288

289

290

291

292

293

294

295

296

297

298

299

300

301

302

303

retroversion. J. shoulder Elb. Surg. [Internet]. 2006 Jul;15(4):521-6. Available from: http://www.ncbi.nlm.nih.gov/pubmed/16831661doi:10.1016/j.jse.2005.10.003

6. Ganapathi A, McCarron JA, Chen X, Iannotti JP. Predicting normal glenoid version from the pathologic scapula: A comparison of 4 methods in 2- and 3-dimensional models. J. Shoulder Elb. Surg. [Internet]. 2011;20(2):234-244. Available from:

http://dx.doi.org/10.1016/j.jse.2010.05.024doi:10.1016/j.jse.2010.05.024

7. Hasan SS, Leith JM, Campbell B, Kapil R, Smith KL, Matsen FA. Characteristics of unsatisfactory shoulder arthroplasties. J. shoulder Elb. Surg. [Internet]. 2002 Sep;11(5):431-41. Available from: http://www.ncbi.nlm.nih.gov/pubmed/12378161doi:10.1067/mse.2002.125806

8. Hendel MD, Bryan JA, Barsoum WK, Rodriguez EJ, Brems JJ, Evans PJ, et al. Comparison of Patient-Specific Instruments with Standard Surgical Instruments in Determining Glenoid Component Position. J. Bone Jt. Surgery-American Vol. [Internet]. 2012;94(23):2167-2175. Available from: http://content.wkhealth.com/linkback/openurl?sid=WKPTLP:landingpage\&an=00004623201212050-00010doi:10.2106/JBJS.K.01209

9. Hoenecke HR, Hermida JC, Flores-Hernandez C, D’Lima DD. Accuracy of CT-based measurements of glenoid version for total shoulder arthroplasty. J. shoulder Elb. Surg. [Internet]. 2010 Mar;19(2):166-71. Available from: 
Glenoid prediction using statistical shape modeling

http://www.ncbi.nlm.nih.gov/pubmed/19959378doi:10.1016/j.jse.2009.08.009

305

306

307

308

309

310

10. Iannotti JP, Jun B-J, Patterson TE, Ricchetti ET. Quantitative Measurement of Osseous Pathology in Advanced Glenohumeral Osteoarthritis. J. Bone Jt. Surg. [Internet]. 2017;99(17):1460-1468. Available from: http://insights.ovid.com/crossref?an=00004623201709060-00006doi:10.2106/JBJS.16.00869

11. Iannotti JP, Weiner S, Rodriguez E, Subhas N, Patterson TE, Jun BJ, et al. ThreeDimensional Imaging and Templating Improve Glenoid Implant Positioning. J. Bone Jt. Surgery-American Vol. [Internet]. 2015;97(8):651-658. Available from: http://content.wkhealth.com/linkback/openurl?sid=WKPTLP:landingpage\&an=00004623201504150-00006doi:10.2106/JBJS.N.00493

12. Levy JC, Everding NG, Frankle MA, Keppler LJ. Accuracy of patient-specific guided glenoid baseplate positioning for reverse shoulder arthroplasty. J. Shoulder Elb. Surg. [Internet]. 2014;23(10):1563-1567. Available from:

http://dx.doi.org/10.1016/j.jse.2014.01.051doi:10.1016/j.jse.2014.01.051

13. Lewis GS, Armstrong AD. Glenoid spherical orientation and version. J. shoulder Elb. Surg. [Internet]. 2011 Jan;20(1):3-11. Available from: http://www.ncbi.nlm.nih.gov/pubmed/20932782doi:10.1016/j.jse.2010.05.012 
Glenoid prediction using statistical shape modeling

for PCA based statistical models. Insight J. 2012;1:1-18.

323 15. Matsen FA, Clinton J, Lynch J, Bertelsen A, Richardson ML. Glenoid component failure in total shoulder arthroplasty. J. Bone Joint Surg. Am. [Internet]. 2008 Apr;90(4):885-96. Available from: http://www.ncbi.nlm.nih.gov/pubmed/18381328doi:10.2106/JBJS.G.01263

16. Moineau G, Levigne C, Boileau P, Young A, Walch G, French Society for Shoulder \& Elbow (SOFEC). Three-dimensional measurement method of arthritic glenoid cavity morphology: feasibility and reproducibility. Orthop. Traumatol. Surg. Res. [Internet]. 2012 Oct;98(6 Suppl):S139-45. Available from: http:/www.ncbi.nlm.nih.gov/pubmed/22964089doi:10.1016/j.otsr.2012.06.007

17. Mullaji AB, Beddow FH, Lamb GH. CT measurement of glenoid erosion in arthritis. J. Bone Joint Surg. Br. [Internet]. 1994 May;76(3):384-8. Available from: http://www.ncbi.nlm.nih.gov/pubmed/8175838

335 18. Mutsvangwa T, Burdin V, Schwartz C, Roux C. An automated statistical shape model developmental pipeline: application to the human scapula and humerus. IEEE Trans. Biomed. Eng. [Internet]. 2014;62(4):1-1. Available from: http://ieeexplore.ieee.org/lpdocs/epic03/wrapper.htm?arnumber=6949148doi:10.1109/TB ME.2014.2368362 
Glenoid prediction using statistical shape modeling

340 19. Myronenko A, Xubo Song. Point Set Registration: Coherent Point Drift. IEEE Trans. Pattern Anal. Mach. Intell. [Internet]. 2010 Dec;32(12):2262-2275. Available from: http://ieeexplore.ieee.org/document/5432191/doi:10.1109/TPAMI.2010.46

20. Nyffeler RW, Jost B, Pfirrmann CWA, Gerber C. Measurement of glenoid version: Conventional radiographs versus computed tomography scans. J. Shoulder Elb. Surg. 2003;12(5):493-496. doi:10.1016/S1058-2746(03)00181-2

21. de Oliveira ME, Netto LMG, Kistler M, Brandenberger D, Büchler P, Hasler C-C. An image-based method to automatically propagate bony landmarks: application to computational spine biomechanics. Comput. Methods Biomech. Biomed. Engin. [Internet]. 2015 Oct;18(14):1535-42. Available from: http:/www.ncbi.nlm.nih.gov/pubmed/24960066doi:10.1080/10255842.2014.927445

351 22. Plessers K, Vanden Berghe P, Van Dijck C, Wirix-Speetjens R, Debeer P, Jonkers I, et al. Virtual reconstruction of glenoid bone defects using a statistical shape model. J. Shoulder Elb. Surg. [Internet]. 2017;1-7. Available from: https://doi.org/10.1016/j.jse.2017.07.026doi:10.1016/j.jse.2017.07.026

23. Poltaretskyi S, Chaoui J, Mayya M, Hamitouche C, Bercik MJ, Boileau P, et al. Prediction of the pre-morbid 3D anatomy of the proximal humerus based on statistical shape modelling. Bone Joint J. [Internet]. 2017 Jul;99-B(7):927-933. Available from: 
Glenoid prediction using statistical shape modeling

360 24. Rajamani KT, Styner M a, Talib H, Zheng G, Nolte LP, González Ballester M a.

361 Statistical deformable bone models for robust 3D surface extrapolation from sparse data.

362 Med. Image Anal. [Internet]. 2007 Apr [cited 2011 Jul 8];11(2):99-109. Available from:

363 http://www.ncbi.nlm.nih.gov/pubmed/17349939doi:10.1016/j.media.2006.05.001

364 25. Scalise JJ, Codsi MJ, Bryan J, Iannotti JP. The three-dimensional glenoid vault model can 365 estimate normal glenoid version in osteoarthritis. J. Shoulder Elb. Surg. 2008;17(3):487$366 \quad$ 491. doi:10.1016/j.jse.2007.09.006

367 26. Shi L, Griffith JF, Huang J, Wang D. Excellent side-to-side symmetry in glenoid size and 368 shape. Skeletal Radiol. 2013;42(12):1711-1715. doi:10.1007/s00256-013-1728-y

369 27. Terrier a, Ston J, Larrea X, Farron a. Measurements of three-dimensional glenoid erosion 370 when planning the prosthetic replacement of osteoarthritic shoulders. Bone Joint J.

371 [Internet]. 2014 Apr [cited 2014 Oct 27];96-B(4):513-8. Available from:

372 http://www.ncbi.nlm.nih.gov/pubmed/24692620doi:10.1302/0301-620X.96B4.32641 
Glenoid prediction using statistical shape modeling

374 Tables \& Figures

375 Table I - Effect of SSM-based prediction on OA glenoids. Predicted correction of glenoid 376 version, inclination and medialization for the 30 scapulae with OA glenoids. The amount of

377 correction was calculated separately for each Walch classification. Results are reported as mean \pm 378 standard deviation where applicable. Positive (negative) signs corresponds to increase (decrease)

379 of the respective measure in the present OA shape relative to the SSM-based reconstructed 380 premorbid shape. For example, a negative change in version means that the SSM-based prediction 381 corrects the posterior OA wear to a more neutral orientation.

382

\begin{tabular}{ccccc}
\hline & $\mathrm{N}$ & $\begin{array}{c}\Delta \text { version } \\
\left({ }^{\circ}\right)\end{array}$ & $\begin{array}{c}\Delta \text { inclination } \\
\left({ }^{\circ}\right)\end{array}$ & $\begin{array}{c}\Delta \text { medialization } \\
(\mathrm{mm})\end{array}$ \\
\hline A1 & 7 & $1.4 \pm 2.9$ & $2.9 \pm 5.0$ & $-0.2 \pm 1.3$ \\
A2 & 5 & $-6.3 \pm 8.5$ & $2.8 \pm 4.1$ & $0.6 \pm 1.4$ \\
B1 & 5 & $0.7 \pm 1.8$ & $1.0 \pm 2.6$ & $0.1 \pm 0.7$ \\
B2 & 11 & $-8.1 \pm 4.6$ & $1.0 \pm 4.5$ & $-0.8 \pm 0.9$ \\
B3 & 1 & -22.6 & 4.5 & 0.3 \\
D & 1 & 8.9 & -3.9 & 1.0 \\
\hline
\end{tabular}


Glenoid prediction using statistical shape modeling
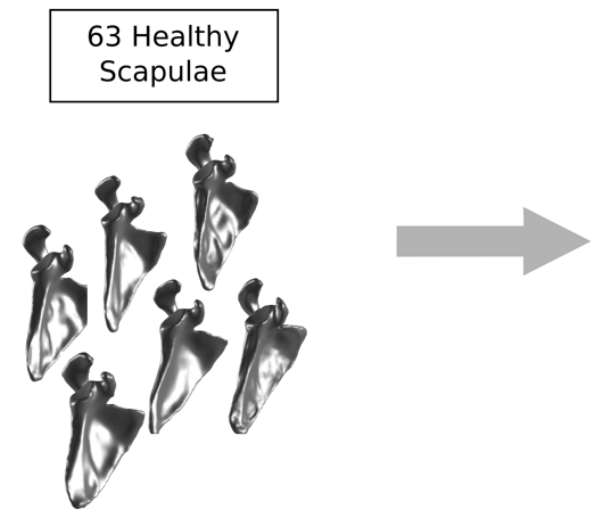

$$
\begin{aligned}
& \text { Glenoid to be } \\
& \text { Reconstructed }
\end{aligned}
$$
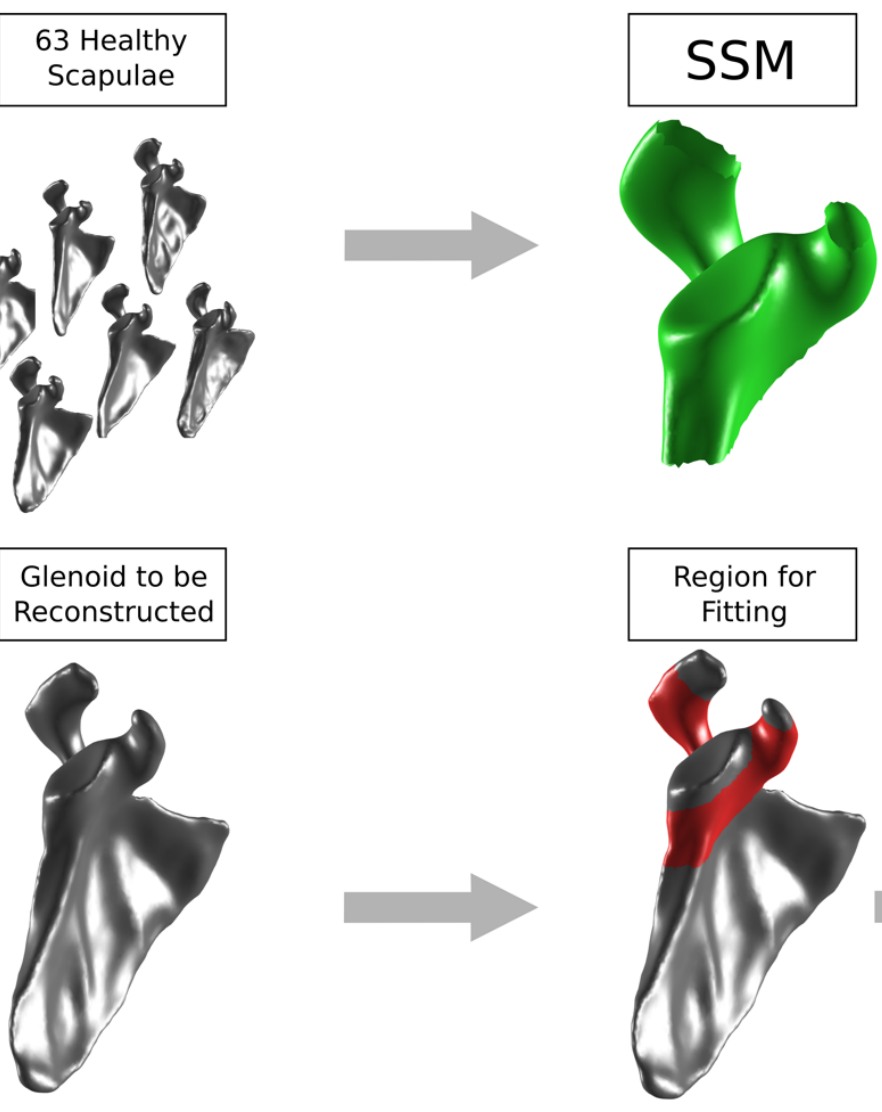

Predicted Glenoid Shape

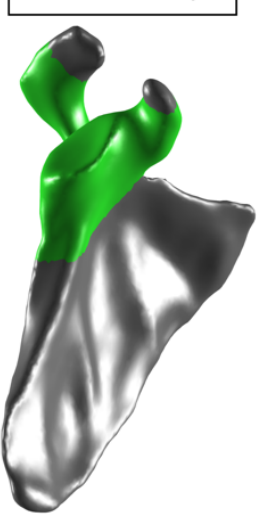

Figure 1 - SSM-based prediction of premorbid glenoid surface: A SSM was built from a population of healthy glenoid surface models. The predicted premorbid glenoid cavity of a specific scapula was obtained by fitting the SSM to the bony region surrounding the glenoid cavity (red area). Based on local fitting, the SSM provided the shape of the healthy glenoid (green area). The performance of the prediction approach was evaluated on healthy scapulae following a leave-oneout strategy. 
Mode 1

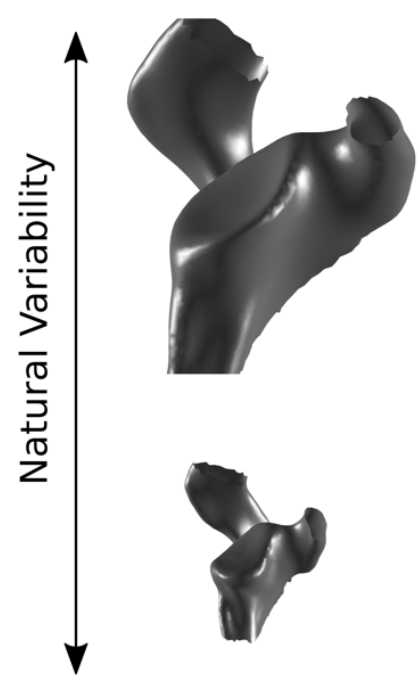

Mode 2
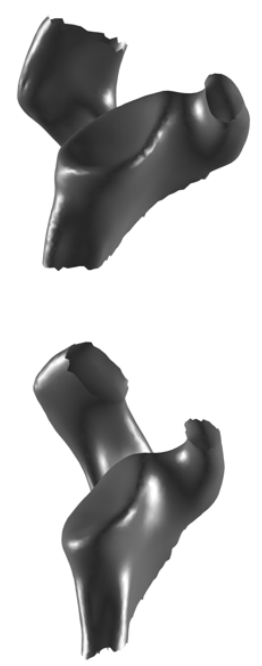

Mode 3
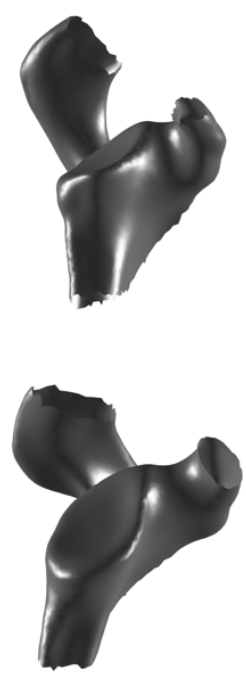

392 Figure 2 - Statistical shape model of healthy scapulae: The three major modes of variation of

393 the SSM constructed from all the training data are represented. These modes account for 394 approximately $40 \%, 13 \%$, and $9 \%$ of the overall variability across subjects, respectively. 
Glenoid prediction using statistical shape modeling

A Mean error across subjects

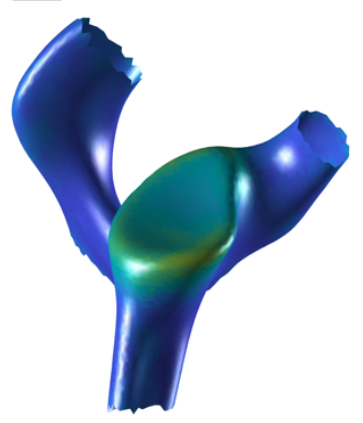

\section{B}

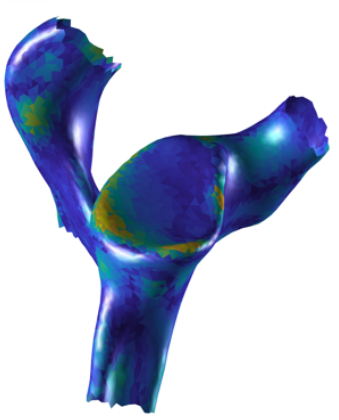

C Largest reconstruction error
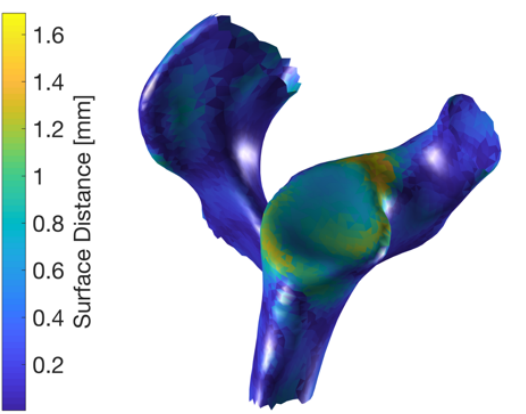

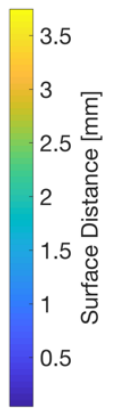

Figure 3 - Surface reconstruction error on healthy scapulae: Pointwise average surface

397 distances between original and reconstructed shapes of the glenoid region. The extent of the

398 surface model was chosen to minimize glenoid reconstruction error. Figure (A) shows the average

399 surface distance across subjects on the mean shape of the healthy scapulae dataset. Figures (B) and

400 (C) show shape and surface distance for the best and worst fitting scapula, respectively. 
A Version

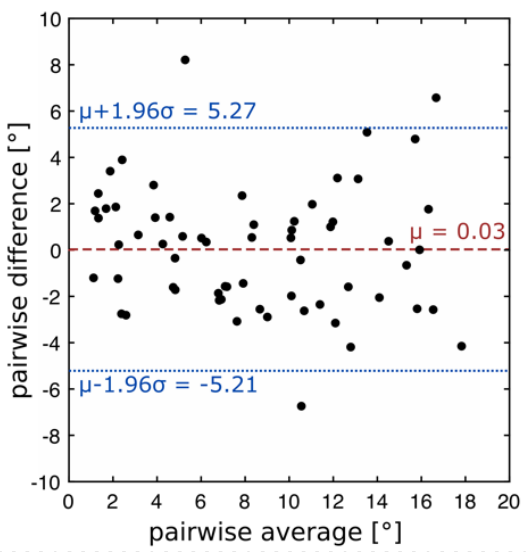

B Inclination

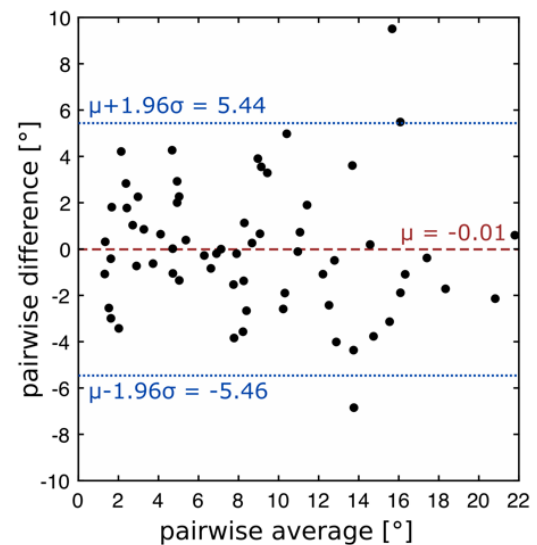

C Medialization

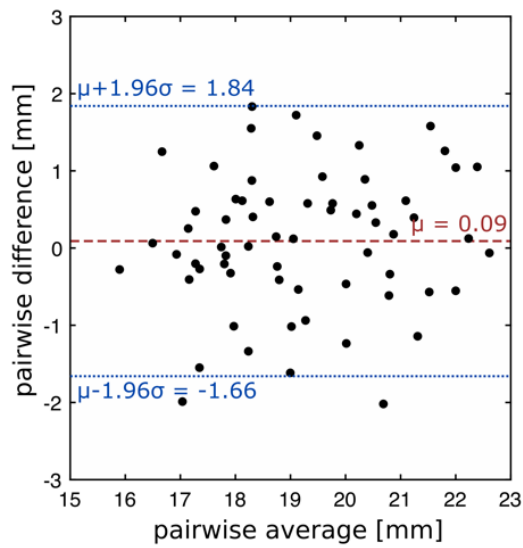

Figure 4 - Glenoid reconstruction error on healthy scapulae: Bland-Altman representations

403 of differences between original and reconstructed glenoid characteristics. Subfigures (A), (B),

404 and (C) show the reconstruction errors between original and predicted glenoid version, inclination,

405 and medialization. The $\mathrm{x}$-axis (y-axis) refers to the average (difference) between original and 406 predicted value of each measurement. Mean and 95\% limits of agreement of the differences

407 between original and predicted measurements are indicated by dashed red and dotted blue lines, 408 respectively. 
Glenoid prediction using statistical shape modeling

A Version $\left[{ }^{\circ}\right]$

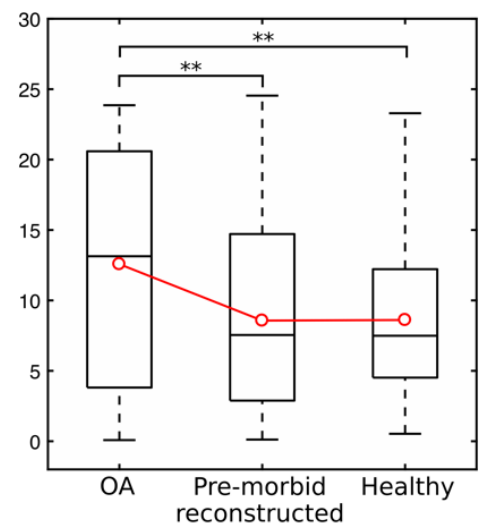

B Inclination $\left[{ }^{\circ}\right]$

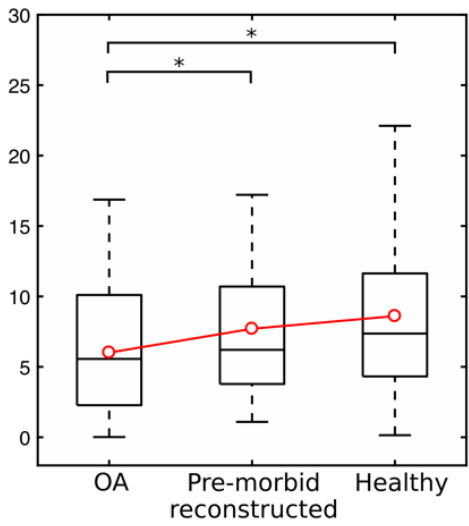

C Medialization [mm]

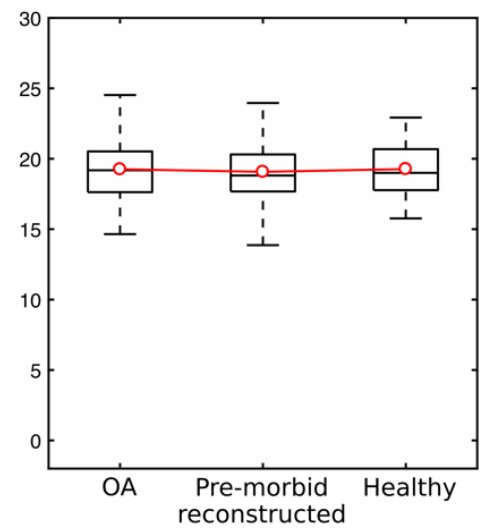

410 Figure 5 - Comparison of glenoid version, inclination and medialization between OA

411 glenoids, the SSM-based reconstructions and the healthy population. Statistically significant

412 differences between groups are indicated by star markers, where ** corresponds to $\mathrm{p}<0.01, *$ to

$413 \mathrm{p}<0.05$ and no marker to $\mathrm{p}>0.05$. Comparisons between groups 'OA' and 'pre-morbid

414 reconstructed' are based on paired Student's t-test; comparisons with 'healthy' on unpaied two

415 sample Student's t test. The red markers indicate the mean of each group. 